\title{
ARNO HOLZ' DAFNIS: DIE PARODIE ALS NATURALISTISCHE KUNST
}

Liest man eine Parodie, dann neigt man allzu leicht dazu, jede schöpferische Ursprünglichkeit oder getreue Umsetzung der Wirklichkeit in dichterische Formen stillschweigend als Leistung des parodierten Dichters zu akzeptieren und dem parodierenden Dichter dagegen nur ein gewisses stilistisches Können zuzuerkennen. Das heißt, der Verfasser der Vorlage erscheint gleichsam als ein Zauberer, der ein Stück Wirklichkeit hervorgezaubert oder wenigstens in Worte gefaßt hat, der Parodist wiederum als ein bloßer Zauberkünstler, als eine Art Taschenspieler. Eine solche Einstellung bringt unweigerlich mit sich, daß der Wirklichkeitsanspruch der in der Parodie dargestellten Welt weitgehend entkräftigt wird. Gerade an diesem Punkt muß es überraschen, einen der größten Parodisten seit dem 18. Jahrhundert zu finden in Arno Holz, der gleichzeitig als der - man denke etwa an Gerhart Hauptmanns Widmung von Vor Sonnenaufgang - , konsequenteste Realist" gilt ${ }^{1}$. Nicht daß es keine anderen Parodisten unter den ,Jüngstdeutschen" gibt: Conrad Alberti schreibt Im Suff! Naturalistische Spitalkatastrophe in zwei Vorgängen (Berlin, 1890) als Parodie auf die Aufführungen der „Freien Bühne” in Berlin $^{2}$, und - um nur noch ein berühmtes Beispiel zu nennen - Otto Erich Hartleben satirisiert die Ibsen-Mode mit Henrik Ipse, Der Frosch. Familiendrama in einem Act. Deutsch von O. E. Hartleben (Leipzig, 1889) ${ }^{3}$. Aber letzten Endes erscheinen solche Parodien als Gelegenheits-Dichtungen, die einer vorübergehenden Anregung entstammten, also nichts Wesentliches über die künstlerischen Absichten und die poetische Entwicklung der Verfasser besagen. Ja, die Mehrzahl der naturalistischen Parodien gehört schlechthin zur wechselseitigen Polemik, die die anfängliche Ziellosigkeit der Naturalisten kennzeichnet ${ }^{4}$. Anders verhält es sich jedoch mit Arno Holz' Parodien.

Überhaupt tritt Holz gewissermaßen erst als Parodist hervor: zusammen mit Johannes Schlaf verleiht er dem Naturalismus eine neue, über Zola hinausgreifende Zielrichtung, als er seinen berühmten Papa Hamlet 1889 unter dem Pseudonym Bjarne P. Holmsen erscheinen läßt ${ }^{5}$. Schon 1885 parodierte er in seinem Buch der Zeit, wohl dem wichtigsten Gedichtsbändchen des Naturalismus, Eichendorff und andere ${ }^{6}$, und 1892 erscheint - gleichsam als letztes Zeugnis ihrer Zusammenarbeit - Der geschundene Pegasus. Eine Mirlitoniade in Versen von Arno Holz und Bildern von Johannes Schlaf (Berlin, 1892), eine Wilhelm Busch-Parodie?. Danach setzt Holz seine parodistischen Dichtungen fort mit der Komödie Die Sozialaristokraten, die einem satirischen Angriff auf die Dichter des Friedrichshagener Kreises diente. In seinen Blechschmieden (Leipzig, 1902) rechnet Holz in einzelnen Parodien mit fast allen mittelmäßigen, zeitgenössischen Schreibern ab. Selbstverständlich können diese Paro- 
dien damit erklärt werden, daß Holz aus der Berühmtheit skandinavischer Dichter Kapital schlagen wollte, oder da $\beta$ fast alle Naturalisten sich überhaupt als Rebellen und Reformer vorkamen und dementsprechend bemüht waren, ihre Vorgänger und ,,rückständigen” Zeitgenossen anzuprangern. Im Fall Holz' kommt außerdem noch dazu, daß er sich zeit seines Lebens als zu Unrecht ignoriert vorkam. Aber weder eine opportunistische noch eine satirische noch eine rein biographische Auslegung erklärt die Entstehung von ,,Des berühmbten Schäffers Dafnis sälbst verfärtigte / unter dem Titel OMNIA MEA fürmahls ans Licht gestellte und von ihme mit einem lästerlichen Nohtwendigen Vorbericht an den guht hertzigen Leser lihderlich verunzihrte / höchst sündhaffte Sämbtliche FreB-Sauff- und Venus-Lieder / vermehrt und verbässert durch vihle biß anhero noch gäntzlich ohngetrukkt gewesene / benebst angekänckten Auffrichtigen und Reue mühtigen Buß-Thränen / vergossen durch den sälben Auctorem / nachdäme dihser mit herein gebrochenem Alters Gebrest auß einem Saulo zu einem Paulo geworden /gesammblet / colligiret / sowie mit einem nüzzlichen Fürvermärck versorgt über die besondre Lebensumbstände des selig Verblichnen / allen Christlichen Gemühtern zu dihnlicher Abschrikkung bekant gegeben / inssondre der schwanckenden Jugend / durch Selamintem. Konstantinopul \& Leipzig / getrukkt in dihsem Jahr". So lautet der Untertitel zu Dafnis: Lyrisches Porträt aus dem 17. Jahrhundert, in dem die parodistische Kunst Holz' ihren Gipfel erreicht ${ }^{8}$. Die Barock-Dichtung zählte kaum zu den modischen Vorbildern, gegen die die Naturalisten oft ins Feld zogen, und wer könnte Opitz, Stieler, Fleming oder Hofmannswaldau zu den gefährlichen Gegnern des Naturalismus rechnen. Man spürt sowieso, daß $\mathrm{Holz}$ keine feindselige, sondern eine geradezu liebevolle, einfühlsame Haltung einnimmt ${ }^{9}$. Und selbst wenn man Erwin Rotermunds Interpretation zustimmt $^{10}$, Holz habe gleichsam als Vorgänger von Wedekind und dem jungen Brecht das Primitive, Lebenslustige, ja sogar Liederliche im Gegensatz zur überzivilisierten Welt des frühen 20. Jahrhunderts verherrlichen wollen - und in dieser Beziehung fand das Buch nicht wenig Anklang unter Holz' Zeitgenossen ${ }^{11}{ }_{-}$, so muß man dennoch zugeben, $\mathrm{da} B$ der Verfasser nur zu einem so durchsichtigen Zweck kaum eine derart durchdachte, sorgfältig aufgebaute Grundlage benötigt hätte ${ }^{12}$. Im folgenden wird auch gezeigt, daß diese groß angelegte Parodie ja geradezu als die notwendige Folge und Verwirklichung der Holzschen Literaturtheorie entstand, daß sie auch als solche verstanden sein muß - vielleicht sogar in einem nicht einmal von Holz selber geahnten Maß -, daß sie aber, so verstanden, die Grenzen der lyrischen Parodie sprengt.

Immer wieder betont $\mathrm{Holz}$ in der letzten Fassung seiner theoretischen Schriften, er habe zusammen mit Johannes Schlaf das sogenannte naturalistische Drama, überhaupt das moderne Drama eingeführt. Wie Holz öfter unterstreicht, liegt das Dramatische im Charakter, nicht in der Handlung: ,Die Menschen auf der Bühne sind nicht der Handlung wegen 
da, sondern die Handlung der Menschen auf der Bühne wegen. Sie ist nicht der Zweck, sondern nur das Mittel" (X, 224). Der Charakter offenbart sich nach Holz in der Sprache der Gestalten, und eigentlich in seiner Wiedergabe der natürlichen Sprache seiner Umwelt sieht Holz seine große Leistung, denn über die neue ,,Methode” im Drama sagt er: „Ich begnüge mich hier nur anzudeuten, wie ihr letztes Fundament darin beruht, daß die uns für das Theater vor allem eine neue Sprache gegeben: die unmittelbar lebendige, statt der bisher konventionell überliefert gewesenen literarisch toten" $(X, 590)$. Die genaue Reproduktion der natürlichen, modernen Sprache der Gestalten kennzeichnet demzufolge das von Holz und Schlaf gemeinsam geschaffene, gerade deshalb von dem alten Fontane als ,Neuland" 13 beschriebene Drama Die Familie Selicke, das in die Literaturgeschichte eingegangen ist als das am strengsten naturalistische Kunstwerk ${ }^{14}$. Die Verwandtschaft $z$ wischen dem Drama und dem lyrischen ,Porträt aus dem 17. Jahrhundert” ist klar zu erkennen, eine Verwandtschaft, auf die schon Otto Julius Bierbaum aufmerksam macht: ,Ein Unikum in der That. Eine Kuriosität sonder Gleichen. Echte, wirkliche Gedichte aus dem siebenzehnten Jahrhundert, - und der lyrische Revolutionär unserer Zeit hat sie geschrieben. Wie hat sich dieses Wunder begeben? Die Antwort klingt paradox genug: Mit den Mitteln der naturalistischen Technik. Nämlich so: wie der Dichter der ,Familie Selike" [sic!] die Sprache seiner Personen aus genau registrirten Wendungen der lebendigen heutigen Sprache ihres Milieus zusammengesetzt hat, so hat der Dichter der Lieder auf einer alten Laute die lyrische Sprache seines Dafnis mosaikartig aus thatsächlich vorhandenen, von Dichtern des siebenzehnten Jahrhunderts benützten Wendungen zusammengesetzt. Sein Werk ist also eigentlich nur das der Komposition und man könnte geneigt sein, darüber despektirlich zu urteilen, wenn es nicht eben doch gleichzeitig ein ganz außerordentliches Kunstwerk wäre. Und das ist es wirklich.... Aus einer ganzen Reihe von Dichtern hat er Einen gemacht, seinen Dafnis, und es ist ihm das Unglaubliche gelungen, daß diese zusammengesetzte Figur mit allen Reizen einer ausgeprägten Persönlichkeit wirkt" ${ }^{15}$. Wohl steht auch hier der Charakter im Mittelpunkt, wie Holz es vom Drama erfordert. Wohl leistet Holz in seiner DafnisDichtung genau das für die Sprache des 17. Jahrhunderts noch einmal, was er für die Sprache seiner Berliner Zeitgenossen verrichtete. Aber Holz' Leistung läßt sich keineswegs mit dem Gleichnis eines Mosaiks umschreiben, denn weiter in der Rezension behauptet der Dichter dieser „,Lieder auf einer alten Laute”: ,,Mein Buch ist durchaus original. Nicht blos als Ganzes, sondern auch in seinen Einzelheiten. Es ist mir vollkommen unerfindlich, wie Bierbaum, der sonst so Gescheite, sich die befremdliche Phantasiekonstruktion zurechtbasteln konnte, ,aus einer ganzen Reihe von Dichtern Einen zu machen", läge technisch wie psychologisch überhaupt innerhalb einer Möglichkeit. ... Nicht eine Nachahmung ist mein ,Dafnis”, sondern eine Neuschöpfung; und zwar, ich wiederhole 
es, um so komplizirter, als ihr gesammtes Rohmaterial durch umfangreiches Studium bis ins letzte Detail erst erobert werden mußte". Nicht nur ist die Dafnis-Dichtung keinesfalls eine Montage bestehender Redewendungen, sondern auch ebenso wenig nur eine ,Nachahmung” oder eine bloß stenographische Wiedergabe des Gegebenen ${ }^{\mathbf{1 6}}$.

Die Anwendung seiner Methode auf eine Gestalt des 17. Jahrhunderts beweist eindeutig: hier haben wir nicht mehr mit dem mißverstandenen Naturalismus als einer Art ,,Hintertreppenpoetik” zu tun. Daß Holz sich weigert, sich auf einen bestimmten Stoff einengen zu lassen, geht gerade aus seiner Bemerkung über Zola hervor, ,daß der Naturalismus eine Methode ist, eine Darstellungsart und nicht etwa ,Stoffwahl"' $(X, 271)^{17}$. Dennoch muß das Verhältnis zwischen der Kunst und der Wirklichkeit geklärt werden, wenn Dafnis nicht lediglich als Virtuosenstück oder Mosaik abgetan werden soll - eine Gefahr übrigens, über die seine Dramen wie Die Familie Selicke und Die Sozialaristokraten auch nicht erhaben sind. Der Satz, mit dem Holz dieses Verhältnis und das Wesen der Kunst schlechthin umreißt, lautet: ,Die Kunst hat die Tendenz, die Natur zu sein; sie wird sie nach Maßgabe ihrer Mittel und deren Handhabung" (X, 187; vgl. die frühere, oft mißverstandene Formulierung ,wieder die Natur zu sein . . nach Maßgabe ihrer jedweiligen Reproduktionsbedingungen ...", X, 83). Man könnte etwa noch versucht sein, durch Holz' Verwendung von ,Natur" wieder einmal - trotz der sorgfältigen Neufassung des Gesetzes - den Naturalismus nur im Stofflichen, d.h. in einer Wiedergabe der Natur, zu sehen. Aber so naiv ist Holz keineswegs. Denn er wiederholt gegen jeden Angriff aus dieser Richtung, er habe sich nie eingebildet, die Natur wiedergeben $z u$ können ${ }^{18}$. In seiner algebraischen Formel drückt er das Verhältnis zwischen der Kunst und der Natur aus als $\mathrm{K}=\mathrm{N}-\mathrm{x}$, wobei das , $\mathrm{x}$ " die künstlerischen Mittel bezeichnet. Holz betont entschieden, das , ," könne nie ganz verschwin$\operatorname{den}(\mathrm{X}, 130 \mathrm{f}$.).

Seine Formulierung des Problems durch,,weniger $x$ " läßt überhaupt erkennen, daß die Kunst stets weniger als die Natur bleiben muß. Holz lehnt sich direkt auf gegen die herkömmliche, idealistische Auffassung von der Kunst, wonach sie gleich die Natur plus ,, $\mathrm{x}$ " sein soll, denn eine solche Formulierung würde andeuten, die Natur sei in der Kunst mit enthaiten und ein gewisses Etwas käme hinzu. Für Holz aber ist gerade das Umgekehrte der Fall: die Natur kann nie in ihrer Ganzheit im Kunstwerk existieren. Die Mittel zur Schaffung des Kunstwerks müssen durch ihre Bedingtheit von vornherein etwas aus der Natur ausschließen, d.h., es habe keinen Künstler gegeben, ,,der nicht selig war, wenn es ihm gelang, in sein Werk aus ihr auch nur ein Stäubchen zu retten!" (X, 198). In Dafnis' barocker Lyrik erscheint also nur soviel von der Natur wie die Mittel der Sprache und der Formen erlauben, und gerade der Teil der Natur wäre mit keinen anderen Mitteln wiederherzustellen.

Hier hat man, wenn man es so ausdrücken will, eine negative Poetik: 
nicht die Tragweite, sondern die Grenzen der barocken Form und Sprache betrachtet $\mathrm{Holz}$ als notwendig. Weiterhin räumt er ein, der Künstler könne die Natur nie so darstellen, wie sie ist, sondern nur seine Vorstellung von ihr, und zwar wiederum in dem $\mathrm{Maß}$, wie seine Mittel es zulassen: ,Herr Möller-Bruck verschimpfiert meine Formel $\mathrm{K}=\mathrm{N}-\mathrm{x}$ in , $\mathrm{K}=\mathrm{N}+\mathrm{y}^{\prime}$, indem er,$+\mathrm{y}^{\prime \prime}=$,Vorstellungsbild' setzt, und in seiner Einfalt, um nicht zu sagen Einfältigkeit, merkt er nicht, ahnt er nicht einmal, daß dieses ,Plus', dieses ,Vorstellungsbild', mit meinem $\mathrm{N}$ einfach identisch ist. Als ob schon je ein Mensch irgendein Ding selbst reproduziert hätte und nicht bloB immer sein betreffendes Vorstellungsbild! ... So elegant Sie diesen Blödsinn auch auf den Tisch trumpfen: minus $x$ immer! Und zwar, wie ich bereits einmal sagte:, ,schon aus dem ganz einfachen und, wie man wirklich meinen sollte, bereits für jedes Kind plausiblen Grunde, weil das betreffende Reproduktionsmaterial, das uns Menschen zur Verfügung steht, stets unzulänglich bleiben wird"' (X, 198 f. ${ }^{19}$. Zugleich wird das Kunstwerk - und dies betont Holz mit besonderem Nachdruck - eine Art Selbstdarstellung des Künstlers. Ja, so fängt seine Selbstanzeige von Dafnis an: ,Alle Dichtung ist im letzten Grunde Selbstdarstellung. Diese geschieht entweder direkt oder indirekt". Alles muß in die Selbstdarstellung münden, weil der Künstler nur seine ganz individuelle und zeitbedingte Vorstellung von seinem angeblichen Gegenstand, der Natur, wiedergeben kann. In der Dafnis-Dichtung müssen wir die philologische sowie ideengeschichtliche Echtheit der barocken Elemente weitgehend akzeptieren ${ }^{20}$. Wie Erwin Rotermund gezeigt hat, gibt es dennoch - wie sich auch in einer Parodie gehört - Ungereimtheiten, vor allem in der zu starken Betonung des Erotischen. Solche Akzentverschiebungen können aber nicht nur satirisch ausgelegt werden. Sie sind ebenso unerläßlich für die Selbstdarstellung der Dafnis-Gestalt, deren Dichtung ja ein ,lyrisches Porträt” abgeben soll, nicht lediglich eine Montage barocker Redewendungen und Vorstellungen. Innerhalb der Grenzen der barocken Form und Sprache muß das Individuelle durchschimmern. Zugleich will Holz das Menschentypische, das Überzeitliche dieser Gestalt abbilden: ,Mein Individuum ist ein Exemplar jenes alten carpe diem-Typs, der in den verschiedensten Verkleidungen immer wieder aus den Kulturen aller Völker aufgetaucht ist: ,Morgen leben wir nicht mehr, also laßt uns heute leben!' Dieser Typ - ,ewig', wie der des Hamlet oder des Don Quixote - hat zwar bereits Kunst über Kunst selbst produziert, ist aber durch die Mittel dieser noch nie festgehalten worden" 21 . Wieder einmal will Holz also die Grenzen eines mißverstandenen Naturalismus als einer Wiedergabe zeitbedingter Wirklichkeitsbeziehungen sprengen. Doch soll seine Bildung eines ,,ewigen” Typs nicht besonders überraschen, denn gerade sein Zeugnis eines, ,konsequenten Realismus" Papa Hamlet parodiert den weltschmerzlerischen HamletTyp des 19. Jahrhunderts ${ }^{22}$.

Holz sagt, die Selbstdarstellung im Kunstwerk geschehe direkt oder 
indirekt, unabhängig vom Willen des Dichters. Da die Selbstdarstellung in der Lyrik ,,reiner" (d.h. unmittelbarer) stattfindet, gibt er ihr den Vorrang vor dem Drama. In dem von Holz behaupteten Vorrang des Lyrischen sehen wir gleichzeitig eine Rechtfertigung der lyrischen DafnisDichtung als Selbstporträt einer Zeit und eines Menschen dieser Zeit (X, 649). Vorausgesetzt, Holz will nicht über einen Menschen der Zeit schreiben, sondern diesen Menschen sich selber gestalten lassen, dann bietet die Lyrik die beste Möglichkeit gerade, weil das ,, $x$ " als Mittel und Vorstellungsbild in ihr am stärksten zu spüren ist. Auf der anderen Seite schwindet das etwa auf Holz zurückzuführende , $\mathrm{x}$ " dementsprechend, denn auf uns wirken die angeblich von Dafnis' Feder stammenden Lieder in ihrer Ganzheit eher wie ein unmittelbarer, dramatischer Vorgang. Und, wie wir in seiner Selbstanzeige merken, ist diese Wirkung von Holz nicht ganz unbeabsichtigt: ,Im vorliegenden Werk ist zum ersten Mal versucht worden, die lyrische Form der dramatischen adäquat zu handhaben". Hierin bleibt Holz auch konsequent, denken wir doch an seine anderen Werke - vor allem an die mit Schlaf geschaffenen Studien wie „Die papierne Passion" -, in denen die Eliminierung des ,x" einer zunehmenden Dramatisierung gleichzusetzen ist. Die Lieder des Dafnis funktionieren als Selbstdarstellung. Sie beanspruchen unsere Gläubigkeit auf etwa dem gleichen Niveau wie bestimmte Monologe, Monologe, die nicht hauptsächlich als Mitteilungen eines Sachverhalts verstanden werden wollen, sondern als Selbstdarstellungen des Sprechers und dessen intimster, oft von ihm selbst ungeahnter Gedanken. Damit bestätigt sich die von Holz für andere Werke beanspruchte Einheit von der Lyrik und dem Drama (s. X, 473 über Sonnenfinsternis und Ignorabimus), denn die Dafnis-Lieder bilden eine Art ausgedehnten Monolog, ja, sogar ein Monodrama. Und als Monodrama unterliegt die Dafnis-Dichtung fast denselben Gesetzen wie das naturalistische Drama überhaupt.

In seiner zweiten Rezension von Ibsens Gespenster hebt der alte Theodor Fontane mit Recht hervor, daß die naturalistische Kunst auf der ,Überzeugung" beruhe, nicht unbedingt auf einer zeitlosen Wahrheit ${ }^{23}$. Im naturalistischen Drama stammt die von Fontane charakterisierte ,, Macht der Überzeugung" weitgehend von der Milieuschilderung, zu der Holz sich bekennt mit der Behauptung, Menschen ohne Milieu könne er nicht gebrauchen $(X, 232)$. Gestaltet Holz auch wirklich den überzeitlichen Typ des wahren carpe-diem-Menschen, so gilt diese Perspektive nicht weniger für Dafnis als für irgendeinen Charakter in der Familie Selicke, nur daß die Bewohner der Berliner Mietskaserne nie die Dimensionen des barokken Dichters erreichen und deshalb in ihr Milieu tiefer verstrickt zu sein scheinen. Dafnis' Milieu ist ebenso überzeugend wie die rekonstruierte, zugleich als typisch erdichtete Umgebung von Eduard Selicke. Denn wir hören ja von Dafnis' Saufkompanen und von seinen Bettgefährtinnen, von der Kneipe, Kirche und sogar von der Küche: 


Chloe/ geuß uns Koffe ein/
der erfreut itzt mehr denn Wein/
zu gebakknem Lamms-Geschlinge
machen sich itzt Pfifferlinge!
Butter-Milch mit Bayrisch-Kraut
schafft uns nicht zu grohbe Haut/
freundlich reichen wir einander
blau gekochten Bley und Zander. (II, 134)

Eine genauere Differenzierung der Speisen eines Schlemmers im 17. Jahrhundert - er bleibt seiner hedonistischen Parole ,carpe diem” bestimmt treu - könnte man sich kaum wünschen. Die naturalistische Wahrheit ist eine quantitative, denn ein naturalistisches Kunstwerk ist in dem Maß ,,wahr", wie es die ,komplette" Wahrheit bietet ${ }^{24}$. Möglichst wenig wird der Phantasie oder einem zufälligen Mißverständnis des Publikums überlassen, wie wir z.B. an den peinlich detaillierten Bühnenbildern und Regieanweisungen naturalistischer Dramen erkennen. In einem Anhang läßt Holz mit gleicher Akribie seinen fiktiven Dichter selber all die Anspielungen - sei es auf die mythologischen Gestalten, sei es auf die zeitgenössischen Dichter wie Opitz und Fleming - von seiner persönlichen und zeitbedingten Perspektive aus erklären. Das historische Milieu des begabten Genußsuchers vervollständigt Holz durch die Miteinbeziehung eines fiktiven zeitgenössischen Herausgebers für die 13 ,,aufrichtigen und reuemütigen Buß-Tränen". Der Herausgeber kommentiert diese und die vorigen Lieder des nunmehr verstorbenen Dafnis, und durch seine Kommentare wird Dafnis' Umwelt von einer Seite wirksam, die Dafnis verschlossen geblieben war.

Die Rolle des Herausgebers ist jedoch mehr als ein geistreicher Einfall, der die Zeit und deren Urteil über Dafnis vergegenwärtigt. Sie gehört zum notwendigen Abschluß der angedeuteten Handlung. In den Freß-, Saufund Venus-Liedern selbst erfahren wir nämlich, daß der Dafnis zwar Theologie studiert, daß er sein Studium aber gleichsam verfrißt und versäuft - von anderem ganz zu schweigen. Dennoch erweist sich das Studierte als wirksam (eine naturalistische Gestalt kann ihre Vergangenheit nie ganz überwinden), denn im ersten Teil nimmt der Student, der sich immer wieder einen „Madensack" nennt, das alte, für das Barock kennzeichnende, religiöse Motiv des memento mori immer wieder auf. Hiermit legt Holz das Fundament für den sich im 2. Teil entfaltenden Konflikt zwischen Dafnis' Glauben an seine eigene Vergänglichkeit, an einen unvermeidlichen Gerichtstag und seinem Prahlen mit seiner sündhaften Vergangenheit, zwischen seinem Bußbedürfnis und seiner anhaltenden Lebenslust-besser gesagt, seiner Venus-Lust. Der zweite Teil muß von einem anderen Menschen herausgegeben werden, denn darin erleben wir in Dafnis' Gedichten seinen verlorenen Kampf gegen seine Triebe. Nicht einmal sein zunehmendes Alter und seine entsprechende Gebrechlichkeit können seine Lebenslust auf die Dauer dämpfen. Charakteristisch für seine unwandelbare Natur scheint das vorletzte Gedicht, ,Er spührt 
ihn [den Tod] kommen", kann er doch beim anbrechenden Frühling auch im Sterbebett von der Liebe auf der Wiese singen. Und selbst in dem letzten Gedicht, ,Er spricht noch auß dem Grabe”, bleibt er der carpe diem-Typ, dessen letzte Worte sind:

\author{
Horch drümb/ waß mein Staub dir spricht: \\ So vihl Gold hat Ophir nicht/ \\ alß in ihrem Munde \\ die flüchtige Secunde. \\ O Adame/ o Eve/ \\ Vita somnium breve!
}

Wohl behauptet er zuletzt, das Leben sei nur ein kurzer Traum, aber die sonst zu erwartende Reue bleibt aus. Die Niederlage, die er in seinem zeittypischen, wenn nicht geradezu fanatisch geführten Kampf gegen seine Triebe erlitt, hätte er selbst nie bekannt gemacht. Dazu ist er zu sehr in sein Milieu verstrickt. Daß er sich zwischen dem Diesseits und Jenseits hin- und hergezogen fühlt, macht ihn zum typischen Vertreter seiner Zeit. $\mathrm{Da} ß$ er letzten Endes dem Diesseits verfällt, macht ihn vielleicht nicht ganz außergewöhnlich unter allen Menschen des 17. Jahrhunderts, es macht aber sein dichterisches Zeugnis von seinem ,Fall” wohl einmalig, denn zu der Zeit hätte man kaum diese Einstellung als seinen letzten Willen veröffentlicht. Wiederum wird die Dichtung von Dafnis aus diesem Grund zu einem ,,überzeugenden”, dennoch individuellen „Porträt aus dem 17. Jahrhundert", statt nur ein Bild vom 17. Jahrhundert zu bleiben.

Man könnte an diesem Punkt vielleicht einwenden, Holz ist letzten Endes trotz seiner Virtuosität und Quellenkunde doch nicht bedeutend weit über die Poetischen Realisten hinausgekommen. Man denke etwa an die Kostümierungen bei Storm, z.B. in Aquis submersus, oder an die historischen Rahmenerzählungen von C. F. Meyer. Haben wir also nur einen Unterschied im Maß oder tatsächlich einen in der Darstellungsart historischer Zusammenhänge? In der Zahl der geschichtlichen Details, von denen die oben besprochenen nur eine bescheidene Kostprobe darstellen, liegt das Neue nicht. Denn wir spüren, die Freß-, Sauf- und Venus-Lieder stehen Holz' Phantasus viel näher als einer historischen oder historisierenden Novelle des Poetischen Realismus. In seinem Phantasus will Holz ein Weltbild des naturwissenschaftlichen Zeitalters bieten $(\mathrm{X}, 650)$, ein Weltbild, das keine Handlung haben darf $(\mathrm{X}, 651)^{25}$. In der Rolle der Titel-Gestalt will Holz den ,Abglanz" spiegeln, den ihm die Welt in die Seele geworfen hat (X, 651). Man könnte fragen, wie weit das von dem entfernt ist, was die Poetischen Realisten bezweckten, gilt doch als ein Kernbegriff der poetisch realistischen Technik die Vorstellung Otto Ludwigs von ,Erinnerungsbildern” als ein Gleichnis für die Kunst ${ }^{26}$. Für Holz dagegen ist alles in der Dafnis-Dichtung noch gegenwärtig, genau so gegenwärtig wie die ganze Geschichte der Menschheit im Sprecher des Phantasus, der wohl die ihm zugedachten Rollen aus ver- 
gangenen Zeiten ausspielen kann, der aber mit gegenwärtiger Sicherheit behauptet: ,Sieben Billionen ... Jahre ... vor meiner Geburt/ war ich eine Schwertlilie". In seiner Person glaubt der Sprecher des Phantasus die vollständige Vor- und Nachgeschichte sowie die geistige Entwicklung der Menschheit zu erkennen und unmittelbar wieder miterleben zu können, eine Vorstellung also von einer unmittelbaren Gegenwart. Nicht durch den Nebel der dazwischen liegenden Jahre sieht er ,Erinnerungsbilder", sondern mit dramatischer Unmittelbarkeit versucht $\mathrm{Holz}$ das , $x$ " der Mittel und des reflektierenden Geistes aufzuheben. Eine ewige Gegenwart kennzeichnet Phantasus und Dafnis, und der Abstand zwischen uns und einer der von Phantasus übernommenen Rollen oder zwischen uns und dem barocken Dichter wird auf ein Minimum reduziert - genauso wie er fast aufgehoben wird, wenn Kopelke oder eine andere Gestalt der naturalistischen Illusionsbühne zu sprechen anfängt.

Es ist die von Holz erzielte dramatische Unmittelbarkeit, die Mittellosigkeit der Selbstdarstellung in der Dafnis-Dichtung, die die neue Darstellungsart über einen bloßen Gradunterschied zur vorangehenden historischen Dichtung erheben sollte. Der Schluß vom Phantasus lautet: „Mein / Staub verstob; / wie ein Stern strahlt mein / Gedächtnis". Holz nennt seinen Phantasus das Weltbild des naturwissenschaftlichen Zeitalters nicht zuletzt, weil in der Phantasus-Gestalt die Erkenntnisse seiner Zeit seine Dichtung prägen (vor allem die Einflüsse von Charles Darwin, Ernst Mach und Ernst Hacckel). In der Dafnis-Dichtung erleben wir, wie das Gedächtnis eines barocken Dichters ebenfalls als Weltbild sich nach den Erkenntnissen und ,,Reproduktionsbedingungen” seiner Zeit gestaltet und noch - gegenwärtig wie ein Stern - zu uns hinüberstrahlt.

\section{University of Michigan}

Ann Arbor

ROY C. COWEN

\section{Anmerkungen}

1. Allerdings lehnt Holz die Bezeichnung „Naturalismus” für sein Werk ab, denn er geht seines Erachtens über Zola hinaus. Dennoch wird im allgemeinen anerkannt, daß Holz die theoretischen Grundsätze des deutschen Naturalismus festlegt. Zum Beispiel: Bruno Markwardt, Geschichte der deutschen Poetik, Band V: Das zwanzigste Jahrhundert (Berlin, 1967) schreibt: „Aber das prägnante Gesetz naturalistischer Wortkunst suchte und fand erst Arno Holz . . . erst das Zäh-Verbissene, EigenwilligEinseitige und bei aller lockeren Lässigkeit der Darstellung dennoch Zielstrebige im Theoretisieren Arno Holz' drang zum Kernproblem der naturalistischen Gestaltungsmöglichkeit und -notwendigkeit vor" (S. 103).

2. Über seine Absichten äußert sich Alberti in ,Die ,Freie Bühne'. Ein Nekrolog von Conrad Albert (II)", Die Gesellschaft, 6 (1890), 1350 f. Aber ganz offensichtlich gehörte die „Freie Bühne" zu den dauerhaftesten Leistungen des Naturalismus, Albertis Werke dagegen zu den vergänglichsten.

3 Hartlebens ernste Werke leiden aber darunter, daß er sich Sudermann, einen bedeutend schwächeren Dramatiker, zum Vorbild nimmt.

4. Wie unschlüssig die deutschen Naturalisten vor $\mathrm{Holz}$ waren, zeigt neben den schlecht gezielten Parodien von Alberti und Hartleben Karl Bleibtreus einflußreiche Revolution der Litteratur, 2. verbesserte und vermehrte Auflage (Leipzig, 1886), in der 
es heißt: „Die neue Poesie wird vielmehr darin bestehen, Realismus und Romantik derartig zu verschmelzen, dass die naturalistische Wahrheit der trockenen und ausdruckslosen Photographie sich mit der künstlerischen Lebendigkeit idealer Composition verbindet" (S. 31).

5. Obwohl beide Mitarbeiter ihre individuellen Beiträge zunächst als untrennbar darstellen und erst später in einen Streit über ihre gegenseitigen Leistungen geraten, steht es heute ziemlich fest, daß Holz jeweils den Stil bestimmte, Schlaf die Fabel. Eine der jüngsten Untersuchungen bietet Helmut Scheuer, Arno Holz im literarischen Leben des ausgehenden 19. Jahrhunderts (1883-1896): Eine biographische Studie (München, 1971).

6. Alle von Holz allein verfaßten Schriften werden zitiert nach Das Werk, Erste Ausgabe mit Einführungen von Dr. Hans W. Fischer (Berlin, 1924/25). Eine Bibliographie der Einzelausgaben und der Werke mit Oskar Jerschke und Johannes Schlaf findet man bei Scheuer (s. o.).

7. Von allen gemeinsamen Arbeiten und allen Holzschen Parodien ist diese wohl die unbedeutendste.

8. Wie Phantasus unterliegt die Dafnis-Dichtung einer evolutionären Entwicklung. Zuerst erscheint sie als Lieder auf einer alten Laute: Lyrisches Porträt aus dem 17. Jahrhundert (Leipzig, 1903). Erst im folgenden Jahr in München wird sie unter dem Namen Dafnis veröffentlicht und zwar mit neuen Liedern und mit dem Anhang der „BuB-Thränen”. Neue Ausgaben erscheinen 1912, 1918, 1921 und 1922. Hier beschränken wir uns auf die endgültige Fassung im Werk (1924/25).

9. Uberhaupt kehren in Dafnis viele Einstellungen wieder, die Holz bereits im „Widmungsepistel an meine Freunde" im Buch der Zeit als seine eigene ausgibt, z.B. seine carpe diem-Auffassung vom Leben.

10. Erwin Rotermund, Die Parodie in der modernen deutschen Lyrik (München, 1963).

11. In seinem Artikel über die „Parodie” im Reallexikon schreibt Alfred Liede: „Den für Holz seltenen Erfolg verdanken die Lieder . . . nicht der erstaunlichen Kunstfertigkeit, sondern den erotischen Motiven". Es ist vielleicht ein Zeichen der Zeit, daß gerade in den letzten Jahren mehrere Ausgaben des Dafnis erschienen, darunter die Paperback-Edition vom Deutschen Taschenbuch Verlag (München, 1963) (=dtv 96).

12. Daß der Humor dennoch durchaus „modern" im naturalistischen Sinne ist, geht aus dem folgenden Beispiel hervor:

Lohrchen legt sich keusch zu Bett/

plätter alß ein Nudel-Brett.

Wems für der nicht grähst und graut/

stäkkt nicht in der bästen Haut.

Mindestens for dryzehn Groschen

kläbt sie ihr Gesicht voll Moschen;

statt Schänckel hat sie ein paar Staaken/

ihr Näsgen ist ein Feuer-Haaken.

Ihr Bukkel krümmt sich schon vor Gicht/

sie wattscht wie eine Ente;

sie leidets nicht/ sie leidets nicht/

es sey denn ein Studente! (II, 59 f.)

Überhaupt gehört das Studentenhafte zu den Lieblingsthemen des Naturalismus, denkt man doch nicht nur an Holz' und Schlafs Studenten in den Studien um die "Kleine Emmi”, in „Ein Tod", in "Papa Hamlet" und sogar in Die Familie Selicke (Wendt), sondern auch an andere Werke der jungen Generation, z.B. Halbes Jugend. Am glücklichsten verbindet Holz dieses Motiv mit dem Hauptmotiv des Dafnis etwa in $\mathrm{II}, 4 \mathrm{ff}$.

13. Theodor Fontane, Werke. Hrsg. von Edgar Gross. Band XXII/2 (München, 1964), S. 731.

14. Gernot Schley, Die Freie Bühne in Berlin (Berlin, 1967), behauptet sogar: ,.. . so ließ nun die Aufführung der, Familie Selicke' deutlich erkennen, daß die letzte Konsequenz dieses Bühnen-Naturalismus zugleich auch sein Ende ist"' (S. 68).

15. Zitiert von Holz in seiner Selbstanzeige, Die Zukunft, 48 (1904), 489-494.

16. Daß diese Auffassung längst überwunden ist, bezeugt z.B. Wolfgang Kayser, Geschichte des deutschen Verses (Bern/München, 1960), S. $141 \mathrm{f}$.

17. Auch andere Dichter haben versucht, die naturalistische Methode auf einen 
historischen Stoff anzuwenden, darunter Wilhelm Weigand und Gerhart Hauptmann auf Florian Geyers Teilnahme am Bauernkrieg und Hauptmann auf die WeberRevolte. Besonders aufschlußreich scheint in diesem Zusammenhang die Beobachtung von Hans Schwab-Felisch, Gerhart Hauptmann: Die Weber. Dichtung und Wirklichkeit, Ullstein-Buch Nr. 5001 (Frankfurt, 1959): „,Die Weber' als ein vollendetes Kunstwerk im Sinne der Mimese, entsprechen in einer nicht übertroffenen Weise dem Arno Holz'schen Kernsatz zur Theorie des Naturalismus" (S. 111). Wegen der formalen Unterschiede existieren nur begrenzte Parallelen zwischen der Dafnis-Dichtung und diesen Dramen, deren Einordnung in den Naturalismus keineswegs so befremdend wirkt, wie die des , lyrischen Porträts aus dem 17. Jahrhundert". Und letzten Endes zeigen sie kaum mehr von den Implikationen der Holzschen Theorien als Die Familie Selicke.

18. Hier unterscheidet sich Holz seiner Meinung nach von Zola und Taine (X, 364).

19. In diesem Zusammenhang verstehen wir die von Holz beanspruchte Universalität seines Kunstgesetzes: „Das Gesetz, das ich gefunden, begreift ein altes japanisches Götzenbild nicht minder, als eine moderne französische Porträtstatue, einen Böcklin, nicht minder als einen Menzel" (X, 159). Alle Kunstwerke haben gemeinsam - und werden immer gemeinsam haben -, daß deren Mittel sich stets vom Material des wirklichen Gegenstandes unterscheiden, und daß der Mensch die Welt nie so begreifen kann, wie sie wirklich ist.

20. Auf das Problem, es gäbe keine Belege aus dem 17. Jahrhundert für seine Rechtschreibung und für einige Wörter, geht Holz in seiner Selbstanzeige ein. Hier sei auch auf die später zu erörternde, von Fontane bemerkte „Macht der Überzeugung” als Grundprinzip des radikalen Realismus hingewiesen. Wir haben Menschen wie Eduard Selicke sprechen hören, wir haben Dichter aus dem 17. Jahrhundert gelesen, und wir sind überzeugt, sie hätten solche Worte wie in der Familie Selicke und Dafnis benutzen können - und unter den Umständen gebrauchen müssen.

21. In der Phrase „Kunst über Kunst” erkennen wir auch, daß das „, schwindet, daß es aber bei der Autorenangabe wiederkehrt. Hätte Holz jedoch nicht als Verfasser unterzeichnet, dann wäre die Dafnis-Dichtung lediglich eine virtuosenhafte Fälschung, keine Kunst.

22. Es sei auf die Interpretation von Papa Hamlet hingewiesen in Roy C. Cowen, Naturalismus: Kommentar zu einer Epoche (München, 1973), S. 147-156.

23. Theodor Fontane, Werke. Hrsg. von Edgar Gross. Band XXII/2 (München, 1964), S. 708.

24. Schon Zola behauptete, er wolle den experimentalen Roman schreiben ,pour arriver à la connaissance complète d'une vérité" (Les OEuvres Complètes, XLI [Paris, 1928], S. 19).

25. Zum Phantasus: Ingrid Strohschneider-Kohrs, „Sprache und Wirklichkeit bei Arno Holz", Poetica, 1 (1967), 44-66, Gerhard Schmidt-Henkel, Mythos und Dichtung (Bad Homburg/Berlin/Zürich, 1967), S. 132-155 und Roy C. Cowen, a.a.O., S. 223-234. Ausführliche bibliographische Angaben sowie eine lange Einleitung zur ersten Fassung findet man in Arno Holz: Phantasus. Hrsg. von Jost Hermand (New York/London, 1968) (= Johnson Reprint).

26. Siehe Bruno Markwardt, Geschichte der deutschen Poetik. Band IV: Das neunzehnte Jahrhundert (Berlin, 1959), S. 294. 